\title{
HERV-K and HERV-W transcriptional activity in myalgic encephalomyelitis/chronic fatigue syndrome
}

\author{
Lucas S. Rodrigues ${ }^{1 \dagger}$, Luiz H. da Silva Nali ${ }^{1,2 \dagger}$, Cibele O. D. Leal ${ }^{1}$, Ester C. Sabino ${ }^{1,3}$, Eliana M. Lacerda ${ }^{4}$, \\ Caroline C. Kingdon ${ }^{4}$, Luis Nacul ${ }^{4}$ and Camila M. Romano ${ }^{1,5^{*}}$ (C)
}

\begin{abstract}
Background: Chronic fatigue syndrome/myalgic encephalomyelitis (CFS/MS) is an incapacitating chronic disease that dramatically compromise the life quality. The CFS/ME pathogenesis is multifactorial, and it is believed that immunological, metabolic and environmental factors play a role. It is well documented an increased activity of Human endogenous retroviruses (HERVs) from different families in autoimmune and neurological diseases, making these elements good candidates for biomarkers or even triggers for such diseases.

Methods: Here the expression of Endogenous retroviruses K and W (HERV-K and HERV-W) was determined in blood from moderately and severely affected ME/CFS patients through real time PCR.

Results: HERV-K was overexpressed only in moderately affected individuals but HERV-W showed no difference.

Conclusions: This is the first report about HERV-K differential expression in moderate ME/CFS. Although the relationship between HERVs and ME/CFS has yet to be proven, the observation of this phenomenon deserves further attention.
\end{abstract}

Keywords: Endogenous retroviruses, HERV-W, HERV-K, Myalgic encephalomyelitis, Chronic fatigue

\section{Background}

Myalgic encephalomyelitis/chronic fatigue syndrome $(\mathrm{ME} / \mathrm{CFS})$ is a chronic and debilitating disease with unknown etiology [1]. Affected individuals have compromised motor and cognitive capacities. There is a wide variation in the symptoms of this disease, which include joint pains, mood disturbance, and malaise and worsening of symptoms following minimal physical or mental exertion. More severe symptoms can be also present including extreme exhaustion, severe joint pains with no apparent cause, non-restorative sleep and a range of immune and neurological symptoms. These symptoms

\footnotetext{
*Correspondence: cmromano@usp.br

${ }^{\dagger}$ Lucas S. Rodrigues and Luiz H. da Silva Nali contributed equally to this manuscript

${ }^{1}$ Laboratório de Virologia, Instituto de Medicina Tropical de São Paulo, Universidade de São Paulo, Rua Dr. Enéas de Carvalho Aguiar, 470, São Paulo, SP 05403-000, Brazil

Full list of author information is available at the end of the article
}

may lead to depression and social isolation in the person with ME/CFS [1]. The pathophysiology of the ME/ CFS is not understood and there is no diagnostic biomarker available. There is still controversy over the etiology of the disease; however, it is widely accepted that several immunological alterations are present in $\mathrm{ME} /$ CFS patients [2]. In addition, accumulated evidence for an association of ME/CFS with viral infections also exists and many patients report the onset of their symptoms during or right after a flu-like illness [3]. Thereafter, an unusual autoimmune response against the infection would be responsible for the perpetuation of the ME/CFS symptoms. Viral participation is finally supported by the evidences of clinical benefit of patients treated with valganciclovir [4]. Unfortunately, the absence of large cohort studies that investigate at the molecular level the participation of infectious agents on the ME/CFS pathogenesis impairs our understanding of this disease.

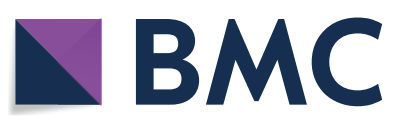

(c) The Author(s) 2019. This article is distributed under the terms of the Creative Commons Attribution 4.0 International License (http://creativecommons.org/licenses/by/4.0/), which permits unrestricted use, distribution, and reproduction in any medium, provided you give appropriate credit to the original author(s) and the source, provide a link to the Creative Commons license, and indicate if changes were made. 
Human endogenous retroviruses (HERVs) are derived from exogenous retroviral infections, which occurred early in the evolution of vertebrates. Due to active replication and transposition events, HERVs are extensively distributed through the host genome and constitute about $8 \%$ of the human genome [5]. Due to accumulated mutations over the primate and human evolution, most HERVs are non-functional, but intact open reading frames of some HERVs persist and can be reactivated in response to systemic and environmental factors such as hormones, stress, and infection by exogenous viruses including almost all human herpesviruses, HIV and others $[6,7]$. Given their potential pathogenic effects, which include molecular mimicry and immune deregulation, HERVs are often postulated as possible causes of autoimmune diseases. Among the more than 30 families, the $\mathrm{K}$ and W families are the most recently integrated, the most active, and have been frequently associated with neurological and autoimmune diseases such as multiple sclerosis, diabetes mellitus, systemic lupus erythematosus, amyotrophic lateral sclerosis and rheumatoid arthritis [8].

To our knowledge, only two studies have investigated the participation of endogenous retroviruses in ME/CFS with contrasting results $[9,10]$.

Given the extensively described altered patterns of HERVs in several diseases and the gap in knowledge of its expression in ME/CFS, we investigated the expression of the HERVs $\mathrm{K}$ and $\mathrm{W}$ in patients diagnosed with ME/CFS.

\section{Methods}

\section{Participants}

We used PBMC samples from a hundred patients diagnosed with ME/CFS and stored in the UK ME/CFS Biobank (UKMEB) at the London School of Hygiene and Tropical Medicine in this study. The UKMEB is among the few biorepositories worldwide with advanced storage and linked research infrastructure dedicated to research into ME/CFS [11]. Seventy-five samples were requested from participants diagnosed with moderate fatigue (ME/ CFSm), and 25 from participants with severe fatigue (ME/CFSs). Participants with ME/CFS were defined as moderate or severely affected based on their mobility: those described as severely affected were house-bound or bed-bound, while those described as having mild/ moderate ME/CFS were ambulatory [11]. Samples from 70 healthy controls (also provided by the UKMEB) were included.

\section{RNA extraction and real time PCR}

RNA extraction from the PBMC samples was performed by the Trizol-chloroform method, with $1 \mathrm{ml}$ Trizol and subsequent addition of chloroform to solubilize lipids allowing its removal. The samples were centrifuged at $15,000 \mathrm{rpm}$ for $15 \mathrm{~min}$ and the upper phase containing the RNA was further used. The material was precipitated with Isopropanol $100 \%$ and washed with $75 \%$ ethanol. In both steps the material was centrifuged at $15,000 \mathrm{rpm}$ for $10 \mathrm{~min}$ at $4{ }^{\circ} \mathrm{C}$. After this process, the pellets were dried at room temperature for $10 \mathrm{~min}$, and the RNA was eluted in $45 \mu \mathrm{l}$ of nuclease-free $\mathrm{H}_{2} \mathrm{O}$. The decontamination of remnant DNA was performed using two rounds DNAse treatment (Turbo DNA-Free (Ambion) following the manufacturer's instruction. The absence of DNA was confirmed by real time PCR without reverse transcriptase using primers for HERV-K or HERV-W (see primers description below).

After this procedure, cDNA was synthesized using the High capacity cDNA Reverse Transcription kit (Ambion, USA) according the manufacturer's instructions. Realtime PCRs were performed for the HERV-W, $-\mathrm{K}$ and the endogenous gene using the primers and conditions used previously by Nali et al. [12] using the Sybr Green method. The primers used are described in Table 1.

The cycling conditions for both HERVs detection were: $50{ }^{\circ} \mathrm{C}$ for $2 \mathrm{~min}, 95^{\circ} \mathrm{C}$ for $10 \mathrm{~min}$, followed by 40 cycles of $95{ }^{\circ} \mathrm{C}$ for $15 \mathrm{~s}, 50{ }^{\circ} \mathrm{C}$ for $1 \mathrm{~min}, 60^{\circ} \mathrm{C}$ for $1 \mathrm{~min}$. HERV activity was qualitatively (referred as presence/absence) and quantitatively (level of expression) evaluated. As positive controls we used a plasmid containing both HERV-W envelope and HERV-K polymerase fragments correspondent to the region covered by the primers used. The level of expression was determined by calculation of $2^{-\Delta \Delta \mathrm{Ct}}$, and the results were represented as fold changes. Statistical analysis was performed using the Wilcox test in the GraphPad Prism program v.6.04.

Samples were only considered positive for HERVs and included in the analyses if expression of the endogenous control was also detected.

Table 1 Primers used in real-time PCR assays

\begin{tabular}{lll}
\hline Oligo & Sense & Antisense \\
\hline HERV-W & CCAATGCATCAGGTGGGTAAC & GAGGTACCACAGACAAAAAATATTCCT \\
HERV-K & TCCCCTTGGAATACTCCTGTTTT & CATTCCTTGTGGTAAAACTTTCCA \\
GAPDH & ACCCACTCCTCCACCTTTGAC & TGTTGCTGTAGCCAAATTCGTT \\
\hline
\end{tabular}




\section{Results}

General description of individuals included in the study is described in Table 2. As expected, women were 4 times more prevalent than men. There were no statistically significant age differences among the groups $(p=0.927$, corrected Pearson's Chi square). Therefore, we adjusted the control group to the same gender prevalence. HERV-K and W expression were evaluated in ME/CFS patients and healthy controls; and some level of expression of HERV-W was detected in all patients with severe fatigue and in 72/75 ME/CFSm (96\%). HERV-K was also detected in all severe cases but in $65 / 75$ of moderate cases $(86.6 \%)$. The healthy control group was very similar to the moderate group, with $68 / 70$ (97\%) and 60/70 (85.7\%) presenting expression of HERV-W and HERV-K respectively (Table 2 ). Only one patient with moderate fatigue and one control individual had no HERV activity at all. No relation was observed regarding HERV detection and duration of disease.

Regarding to the level of expression (quantitative analysis), real time results revealed that HERV-W did not present significant differences when the healthy controls (HCs) or the two ME/CFS groups were compared between each other (Fig. 1a), i.e. ME/CFSs vs HCs $(\mathrm{p}=0.89), \mathrm{ME} / \mathrm{CFSm}$ vs HCs $(\mathrm{p}=0.77), \mathrm{ME} / \mathrm{CFSs}$ vs ME/ CFSm ( $\mathrm{p}=0.95)$, all patients ME/CFS vs HCs $(\mathrm{p}=0.78)$.

On the other hand, the HERV-K expression differed significantly between ME/CFSm group and the HCs $(\mathrm{p}=0.050)$. HERV-K activity was not distinct between the ME/CFS groups: ME/CFSs vs ME/CFSm $(p=0.12)$, ME/CFS vs HCs $(p=0.17)$. ME/CFSs vs HCs $(p=0.97)$ (Fig. 1b).

\section{Discussion}

The most recognized and widely-used case definitions (Fukuda [13] and Canadian Consensus criteria [1]) are based on self-reported symptoms. Studies of energy metabolism, oxidative stress and immunological alterations in ME/CFS have demonstrated imbalance in all these pathways, but the use of such information for diagnostic purposes is still far from reality.
Here, HERV-K and $\mathrm{W}$ transcripts were detected in all groups investigated, and we found that HERV-K was overexpressed in moderate ME/CFS. It is possible that the immunological, genic expression and metabolic alterations are different according to disease severity.

The interplay between endogenous retroviruses and the immune system is complex. ERVs are part of the host genome and in theory, they are supposed to be recognized as self-antigens and an immune tolerance should be established during the early stages of the organism development [14]. However, HERV products can interact with components of the innate immune system leading to the activation of pro-inflammatory pathways or, in some particular cases, their suppression [15]. The syncytin-2 protein for example, is a product of the ERV-FRD Env gene that has an immunosuppressive role by preventing maternal immune response against the fetus [16]. In a distinct scenario, it was demonstrated using psoriasis model that a pro-inflammatory environment could be able to suppress the expression of repetitive elements, including HERVs [17]. It would be reasonable to suggest that the immunological enhancement seen in more severe ME/CFS works by silencing the HERV transactivation that occurs in moderate cases. Such transactivation could be caused by exogenous viral replication or another as yet unknown factor. In line with this, Montoya and colleagues found a cytokine signature of severity in people with ME/CFS [18]. They demonstrated that from the 17 cytokines related to severity, 13 are pro-inflammatory, and (in addition to the worsening of the symptoms) may cause the reversion of the HERV-K activity to levels similar to those seen in healthy individuals. It may similarly occur with HERV-W, which, despite not being at significant levels, there was a slight decrease in people severely affected by ME/CFS when compared to those who are moderately affected.

Infection has often been considered as a trigger to ME/ CFS. Many patients report that the fatigue began during or short after an episode of infectious disease. A number of pathogens including viruses have been associated with this disease [3]. And, due to its life long persistence and

Table 2 Main characteristics of individuals included in the study

\begin{tabular}{|c|c|c|c|c|c|c|}
\hline \multirow[t]{2}{*}{ Participants (\#) } & \multirow[t]{2}{*}{$\begin{array}{l}\text { Age } \\
\text { Median (max, min) }\end{array}$} & \multicolumn{2}{|c|}{ Gender (\%) } & \multirow[t]{2}{*}{$\begin{array}{l}\text { Time of disease } \\
\text { Median ( } \max , \min )\end{array}$} & \multicolumn{2}{|c|}{$\begin{array}{l}\text { Detection of HERV } \\
\text { activity }^{\mathrm{a}}\end{array}$} \\
\hline & & $M$ & $F$ & & HK & HW \\
\hline ME/CFSs (25) & $49.7(22-59)$ & $24 \%$ & $76 \%$ & $15.3(2.8-40)$ & $100 \%$ & $100 \%$ \\
\hline ME/CFSm (75) & $43.5(18-60)$ & $25.4 \%$ & $74.6 \%$ & $9.5(0.2-33.7)$ & $86.6 \%$ & $96 \%$ \\
\hline Controls (70) & $43.8(18-60)$ & $25.7 \%$ & $74.3 \%$ & - & $85.7 \%$ & $97 \%$ \\
\hline
\end{tabular}

a Qualitatively 
(A)

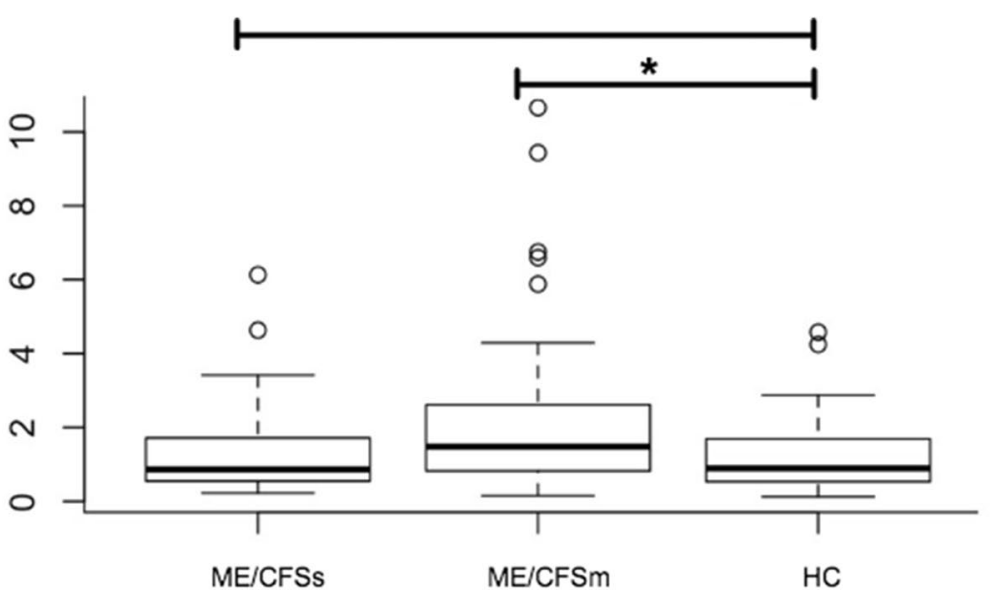

(B)

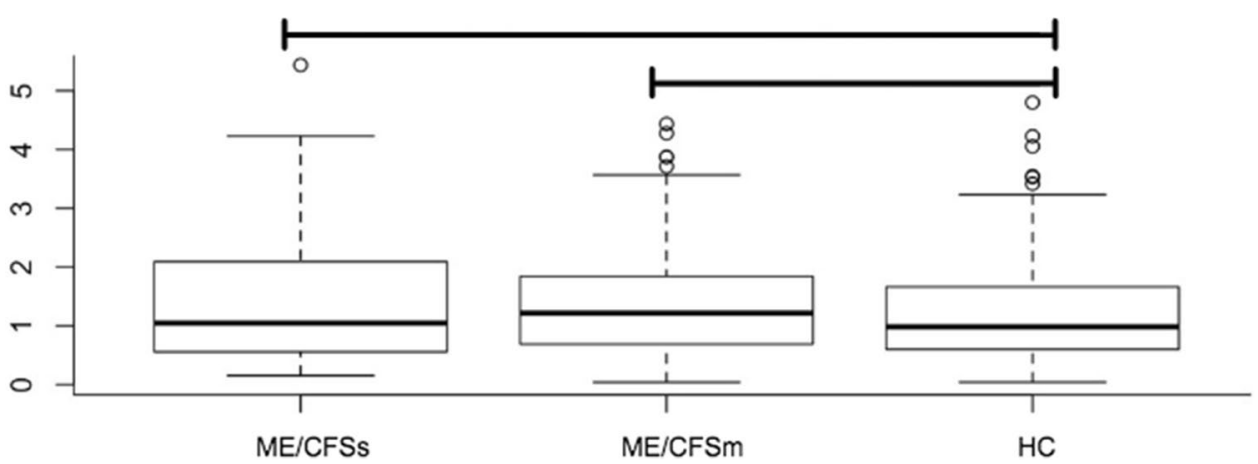

Fig. 1 Boxplot of expression levels (in fold change) of HERVs among the groups. a HERV-K and $\mathbf{b}$ HERV-W. Significance between the groups (obtained by Wilcox test) is evidenced by an asterisk

broad cell tropism, the herpesviridae family, particularly HHV-6 has been considered to be a possible trigger for ME/CFS for many, even though such relationship has not been consistent [3, 9]. Interestingly, HHV-6 as some other herpesviruses, is also capable of transactivating HERVs, particularly, HERV-K [6]. Such transactivation may be either direct (through LTR activation by viral products) or indirect (via transcriptional binding factors and cytokines produced by viral replication) $[3,6]$. It is possible that as the disease progresses, whatever the exogenous infection that would have act as the trigger factor is controlled, and consequently, the HERVs transactivation decrease. Unfortunately, we did not perform serological or molecular tests for exogenous viruses.

Two reports of HERV activity in ME/CFS were published some years ago but the results were conflicting. In 2013 Oakes and his team found no difference on the expression of HERV-K18 envelope in people with ME/ CFS when compared with HCs [9]. In the same year De Meirleir and colleagues, using immunohistochemical methods, found immunoreactivity to HERV proteins (HERV-K, HERV-18, HERV-R and HERV-FRD) in dendritic cells of the duodenum of individuals diagnosed with the syndrome [10], suggesting that alterations in endogenous retroviruses expression pattern may occur in ME/CFS. The differences between the results of Oakes and colleagues and ours may be due to the methods used to detect HERV-K. While the present work used generic primers for HERV-K that allow the detection of hundreds of elements from most HML subfamilies the Oakes team searched for the HERV-K 18 envelope transcripts only, using a method specific to this particular element, while neglecting all the remaining proviruses from the $\mathrm{K}$ family. On the other hand, we are unable to determine which $\mathrm{K}$ family proviruses are involved in the differential expression observed.

The molecular method used here to detect HERV-W was also generic and was widely used in several studies that found differential expression of this element in pathological conditions, including in the blood, brain and CSF of multiple sclerosis (MS) patients [19]. Therefore, despite the similarity of a number of symptoms and the strong immunological component of ME/CFS 
and MS, the mechanisms responsible for HERV reactivation in such diseases are likely distinct.

\section{Conclusion}

In conclusion, this is the first report that demonstrates increased expression of an endogenous retrovirus in the blood of individuals with moderate ME/CFS. While the increased expression of these retroelements can't be directly associated to the ME/CFS pathogeny, the observation of this phenomenon cannot be ignored.

\section{Acknowledgements}

Not applicable.

\section{Authors' contributions}

LSR, LHSN and CODL performed the experiments and wrote the draft of the manuscript. ECS participated in the research design and planning. EML and CCK contributed to samples preparation, worked out technical details and critically revised the manuscript. LN and CMR — research design, responsible of overall direction and planning, and wrote the final version of the manuscript. All authors read and approved the final manuscript.

\section{Funding}

This work was supported by Fundação de Amparo à Pesquisa do Estado de São Paulo (FAPESP) [Grant Number 15/05958-3] and by Programa de fomento às atividades de lideranças científicas dos LIMs do Hospital das Clínicas da FMUSP (PROFAPLIM/HCFMUSP) (Grant No. 26/2019). LHSN, EML and CCK have been funded by the National Institutes of Health (NIH USA) under award number [2R01Al103629], however the content is solely the responsibility of the authors and does not necessarily represent the official views of the $\mathrm{NIH}$.

\section{Availability of data and materials}

Not applicable.

Ethics approval and consent to participate

This study was approved by LSHTM and University of São Paulo ethical committees [\#EC.2017.02 and \#2728254 respectively].

\section{Consent for publication}

Not applicable.

\section{Competing interests}

The authors declare that they have no competing interests.

\begin{abstract}
Author details
${ }^{1}$ Laboratório de Virologia, Instituto de Medicina Tropical de São Paulo, Universidade de São Paulo, Rua Dr. Enéas de Carvalho Aguiar, 470, São Paulo, SP 05403-000, Brazil. ${ }^{2}$ Pós-Graduação em Ciências da Saúde, Universidade Santo Amaro, São Paulo, Brazil. ${ }^{3}$ Departamento de Moléstias Infecciosas e Parasitárias, Faculdade de Medicina da Universidade de São Paulo, São Paulo, Brazil. ${ }^{4}$ Department of Clinical Research, Faculty of Infectious and Tropical Diseases, London School of Hygiene and Tropical Medicine, London, UK. ${ }^{5}$ Hospital das Clinicas HCFMUSP (LIM52), Faculdade de Medicina, Universidade de São Paulo, São Paulo, Brazil.
\end{abstract}

Received: 3 September 2019 Accepted: 9 October 2019

Published online: 15 November 2019

\section{References}

1. Carruthers BM, Jain AK, De Meirleir KL, Peterson DL, Klimas NG, Lerner AM, et al. Myalgic encephalomyelitis/chronic fatigue syndrome. J Chronic Fatigue Syndr. 2003;11(1):7-115.
2. Lorusso L, Mikhaylova SV, Capelli E, Ferrari D, Ngonga GK, Ricevuti G. Immunological aspects of chronic fatigue syndrome. Autoimmun Rev. 2009:8(4):287-91.

3. Rasa S, Nora-Krukle Z, Henning N, Eliassen E, Shikova E, Harrer T, et al. Chronic viral infections in myalgic encephalomyelitis/chronic fatigue syndrome (ME/CFS). J Transl Med. 2018;16(1):268.

4. Watt T, Oberfoell S, Balise R, Lunn MR, Kar AK, Merrihew L, et al. Response to valganciclovir in chronic fatigue syndrome patients with human herpesvirus 6 and Epstein-Barr virus IgG antibody titers. J Med Virol. 2012;84(12):1967-74.

5. Weiss RA. The discovery of endogenous retroviruses. Retrovirology. 2006;3(1):67.

6. Chen J, Foroozesh M, Qin Z. Transactivation of human endogenous retroviruses by tumor viruses and their functions in virus-associated malignancies. Oncogenesis. 2019;8(1):6.

7. Nellåker C, Yao Y, Jones-Brando L, Mallet F, Yolken RH, Karlsson H. Transactivation of elements in the human endogenous retrovirus $W$ family by viral infection. Retrovirology. 2006;3(1):44.

8. Gröger V, Cynis H. Human endogenous retroviruses and their putative role in the development of autoimmune disorders such as multiple sclerosis. Front Microbiol. 2018;9:265.

9. Oakes B, Hoagland-Henefield M, Komaroff AL, Erickson JL, Huber BT. Human endogenous retrovirus-k18 superantigen expression and human herpesvirus-6 and human herpesvirus-7 viral loads in chronic fatigue patients. Clin Infect Dis. 2013;56(10):1394-400.

10. De Meirleir KL, Khaiboullina SF, Frémont M, Hulstaert J, Rizvanov AA, Palotás A, et al. Plasmacytoid dendritic cells in the duodenum of individuals diagnosed with myalgic encephalomyelitis are uniquely immunoreactive to antibodies to human endogenous retroviral proteins. In Vivo. 2013;27(2):177-87.

11. Lacerda EM, Mudie K, Kingdon CC, Butterworth JD, O'Boyle S, Nacul L. The UK ME/CFS biobank: a disease-specific biobank for advancing clinical research into myalgic encephalomyelitis/chronic fatigue syndrome. Front Neurol. 2018;9:1026.

12. Nali LHS, Oliveira ACS, Alves DO, Caleiro GS, Nunes CF, Gerhardt D, et al. Expression of human endogenous retrovirus $\mathrm{K}$ and $\mathrm{W}$ in babies. Arch Virol. 2017;162(3):857-61.

13. Fukuda K, Straus SE, Hickie I, Sharpe MC, Dobbins JG, Komaroff A. The chronic fatigue syndrome: a comprehensive approach to its definition and study. International Chronic Fatigue Syndrome Study Group. Ann Intern Med. 1994;121(12):953-9.

14. Tugnet N, Rylance P, Roden D, Trela M, Nelson P. Human endogenous retroviruses (HERVs) and autoimmune rheumatic disease: is there a link? Open Rheumatol J. 2013;7:13-21.

15. Magiorkinis $G$, Hurst TP. Activation of the innate immune response by endogenous retroviruses. J Gen Virol. 2015;96(6):1207-18.

16. Mangeney M, Renard M, Schlecht-Louf G, Bouallaga I, Heidmann O, Letzelter C, et al. Placental syncytins: genetic disjunction between the fusogenic and immunosuppressive activity of retroviral envelope proteins. Proc Natl Acad Sci. 2007;104(51):20534-9.

17. Lättekivi F, Kõks S, Keermann M, Reimann E, Prans E, Abram K, et al. Transcriptional landscape of human endogenous retroviruses (HERVs) and other repetitive elements in psoriatic skin. Sci Rep. 2018;8(1):4358.

18. Montoya JG, Holmes TH, Anderson JN, Maecker HT, RosenbergHasson Y, Valencia IJ, et al. Cytokine signature associated with disease severity in chronic fatigue syndrome patients. Proc Natl Acad Sci. 2017;114(34):E7150-8.

19. Morandi E, Tanasescu R, Tarlinton RE, Constantinescu CS, Zhang W, Tench $C$, et al. The association between human endogenous retroviruses and multiple sclerosis: a systematic review and meta-analysis. PLoS ONE. 2017;12(2):e0172415.

\section{Publisher's Note}

Springer Nature remains neutral with regard to jurisdictional claims in published maps and institutional affiliations. 